\title{
Comparación de la Metodología Mora-Vahrson y el Método Morfométrico para Determinar Áreas Susceptibles a Deslizamientos en la Microcuenca del Río Macho, Costa Rica
}

\author{
A Comparison of the Mora-Vahrson Methodology and the \\ Morphometric Method to Determine Areas Susceptible to \\ Landslides in the Macho River Microbasin, Costa Rica
}

\author{
Adolfo Quesada Román ${ }^{1}$ \\ Universidad de Ginebra, Suiza \\ Sergio Feoli Boraschi ${ }^{2}$ \\ Compañía Nacional de Fuerza y Luz, Costa Rica
}

http://dx.doi.org/10.15359/rgac.61-2.1

\section{RESUMEN}

Se analizan las metodologías Mora-Vahrson (1994) y el método morfométrico para estudiar sus fortalezas y debilidades en la determinación de zonas susceptibles a deslizamientos. Se explican las metodologías, y posteriormente, se hace un estudio de caso en la microcuenca del río Macho al NE de San José para comparar los resultados de ambos métodos. Dicho análisis establece que ambas metodologías son útiles para la determinación de línea base para zonificar áreas susceptibles a deslizamientos, dependiendo de la disponibilidad de los insumos de entrada. Por último, estas herramientas pueden ser usadas para la gestión del riesgo de desastres por procesos de ladera y el ordenamiento territorial.

Palabras clave: Metodología Mora-Vahrson, morfometría, susceptibilidad, deslizamientos, movimientos en masa

1 Geógrafo y Geomorfólogo. Instituto de Ciencias Ambientales, Universidad de Ginebra, Suiza. Correo electrónico: adolfo.quesada@gmail.com

2 Ingeniero Forestal y Gestor de áreas silvestres protegidas. Compañía Nacional de Fuerza y Luz. Correo electrónico: sfeoli@cnfl.go.cr 
Adolfo Quesada Román, Sergio Feoli Boraschi. A Comparison of the Mora-Vahrson Methodology and the Morphometric Method to Determine Areas Susceptible to Landslides in the Macho River Microbasin, Costa Rica

\begin{abstract}
The Mora-Vahrson methodologies (1994) and the morphometric method are analyzed to study their strengths and weaknesses in the identification of areas susceptible to landslides. First, these methodologies are explained; later, a case study is conducted in the Rio Macho microbasin, northeast San Jose, to compare the results of both methods. This analysis establishes that both methodologies are useful for the determination of baseline for zoning areas susceptible to landslides, depending on the availability of inputs. Finally, these tools can be used for disaster risk management by slope processes and territorial planning.
\end{abstract}

Keywords: Methodology Mora-Vahrson, morphometry, susceptibility, landslides, mass movements.

\title{
Introducción
}

Un movimiento en masa o proceso de ladera es el movimiento hacia afuera y debajo de una ladera bajo la influencia de la gravedad, que resulta del fallo de cizallamiento de una sección de la ladera a lo largo de un plano de fractura (Dikau, 2004). Entre los factores que favorecen su desarrollo están el cambio del ángulo de inclinación de una superficie, morfología del terreno, exceso o reducción de carga por obras humanas, deforestación, sismos, agua subterránea, alternancia de humectación-sequedad y congelamiento-deshielo, movimientos tectónicos, actividad volcánica, entre otros (Lugo, 2011).

Los términos para nombrar a los procesos que se dan en las laderas y están regidos por la gravedad derivan del inglés, francés u otros idiomas, la traducción al español a veces genera confusión, por lo que se reconocen varias formas de agrupar estos procesos: procesos de ladera (slope processes), procesos gravitacionales (gravitational processes), procesos de remoción en masa (mass movement processes) o deslizamientos (landslides); todos ellos correctos (Alcántara-Ayala, 2000). Los diferentes procesos de ladera se clasifican, según la clase (WP/WLI 1990; Cruden y Varnes 1996; Alcántara-Ayala 2000; Huggett 2007) en: Deslizamientos, Caídas o Desprendimientos, Volteos, Flujos, Expansión lateral y Movimientos complejos. Los materiales que componen los tipos de procesos de remoción en masa se dividen según el tipo de material en: Roca, Detritos (partículas mayores a $2 \mathrm{~mm}$ ) y Suelos (partículas menores a $2 \mathrm{~mm}$ ).

El conocimiento de los procesos de ladera y su dinámica es importante en la ingeniería, gestión de riesgos, y el ordenamiento territorial; su estudio puede asociarse con movimientos en masa del pasado que podrían reactivarse, la generación de inestabilidad de los taludes por la 
modificación antrópica del uso de la tierra, así como los cambios en los patrones hidrológicos y sísmicos de los terrenos inestables que pueden ser estudiados a partir de las aplicaciones de los Sistemas de Información Geográfica de mediana y alta precisión como el LiDAR (Bierman y Montgomery, 2014). En el caso de este trabajo, se van a explicar, implementar y comparar dos metodologías para la determinación de zonas susceptibles a deslizamientos: la Mora-Vahrson (1994) y la morfometría (Lugo, 1988; Quesada-Román, 2016; Quesada-Román y Barrantes, 2017).

La metodología Mora-Vahrson (MV) es un procedimiento relativamente sencillo y bastante efectivo a la hora de zonificar regiones susceptibles a deslizamientos. Ha sido utilizada en países como Guatemala (Barillas, 2008), El Salvador (SNET, 2004) y Nicaragua (INETER et al., 2004), con buenos resultados, según se desprende de los estudios publicados. En Costa Rica, esta metodología se ha implementado exitosamente en el análisis de la susceptibilidad a deslizamientos en diversas localidades, entre ellas la Península de Papagayo (Mora et al., 2002), El Cacao de Atenas (Mora, 2004a), el cantón de San José (Mora, 2004b), Valle de Orosi (Salazar, 2007) y en el corredor del poliducto entre Siquirres y Turrialba (Segura et al., 2011), por mencionar algunos casos.

La efectividad de la metodología ha sido confirmada en diferentes países, especialmente de América Latina, ejemplo de ello es el trabajo de Barrantes et al. (2011) en un estudio realizado en la microcuenca del río Sarapiquí, en el que se corroboró la coincidencia entre las zonas identificadas como de alta susceptibilidad y los deslizamientos ocurridos el 8 de enero de 2008 a raíz del terremoto de Cinchona. Los resultados fueron suficientemente precisos, en las zonas identificadas como de muy alta y alta susceptibilidad ocurrieron deslizamientos de magnitud considerable. Así mismo, los autores recomiendan que esta metodología se aplique a una escala no mayor que 1:50000, debido a la dificultad de conseguir información detallada de litología, altitud y meteorología.

La morfometría o geomorfometría es el análisis cuantitativo de la superficie terrestre, entre sus variables fundamentales están la altimetría o hipsometría, la pendiente del terreno y la densidad del drenaje; no obstante, se pueden conformar de muchas más, dependiendo de la morfodinámica y morfogénesis (Pedraza, 1996). La geomorfometría puede ser general para estudiar el campo como un continuo, o ser específica para el 
Adolfo Quesada Román, Sergio Feoli Boraschi. A Comparison of the Mora-Vahrson Methodology and the Morphometric Method to Determine Areas Susceptible to Landslides in the Macho River Microbasin, Costa Rica

análisis de las características geométricas y topológicas de las formas del relieve (Evans, 2012). Los análisis morfométricos se pueden dividir en dos partes: uno que estudia las características cuantitativas de las formas (p.e. superficie, altura, pendiente y volumen) y el otro, que se encarga de la investigación de los elementos del relieve a partir de índices numéricos: medios, máximos y mínimos (Lugo, 2011). Los productos de este tipo de análisis son mapas morfométricos, los cuales son útiles en estudios de evolución de laderas, evaluación de erosión y susceptibilidad a peligros (Peña-Monné, 1997).

En el contexto latinoamericano existen muchos ejemplos de la aplicación de métodos morfométricos para la zonificación de procesos de ladera. México es el país donde la geomorfometría ha sido más aplicada en las últimas décadas con una importante producción de tesis de grado, proyectos de investigación y un valioso número de metodologías para determinar áreas susceptibles a procesos de remoción en masa, expuestas en múltiples artículos científicos (Flores y Alcántara, 2002; Borja y Alcántara, 2004; Lugo et al., 2005; Aceves et al., 2006; Borja y Alcántara, 2010; Flores y Alcántara, 2012; Muñiz-Jaúregui y Hernández-Madrigal, 2012; Valerio et al., 2012; Aceves et al., 2014; Galindo y Alcántara, 2014; Legorreta y Lugo, 2014). Otros estudios similares se han realizado en Argentina (Carignano et al., 2014), en Brasil (Marques et al., 2008; Dos Santos et al., 2009), en Costa Rica (Suárez et al., 2009; Quesada-Román, 2016; Quesada-Román y Barrantes, 2017), en Perú (Villacorta et al., 2007), así como en Venezuela (Marcano y Cartaya, 2013; Mujica y Pacheco, 2013), no obstante, generalmente se limitan a la caracterización de la geología y su asociación con la incidencia de estos fenómenos en áreas específicas, sin resultar en una cartografía de áreas susceptibles a procesos de ladera como insumo para la planificación territorial.

\section{Área de estudio}

La cuenca del río Macho es una microcuenca del río Virilla localizada en las coordenadas geográficas -83,927944 a -84,025583 Longitud Oeste y 9,978094 a 10,019968 Latitud Norte en una extensión de $28.39 \mathrm{~km}^{2}$. Se ubica en la ladera suroeste del volcán Irazú en su mayoría en el cantón de Vázquez de Coronado, y en una pequeña proporción en Moravia (Mapa 1). Su origen está determinado por la tectónica regional de Costa Rica, 
que supone un proceso de subducción entre la placa de Cocos y Caribe, así como la deformación intraplaca por distintas estructuras disyuntivas locales o regionales que modelan esta cuenca. Los procesos geológicos alteran lentamente la configuración del espacio geográfico, comparado con las acciones humanas que modifican el medio físico de una manera más acelerada en la escala del tiempo. Los procesos geodinámicos como las erupciones volcánicas y actividad sísmica intervienen en la composición y estabilidad del suelo, además moldean y transforman el relieve. La geología de la microcuenca del río Macho se caracteriza por ser principalmente de origen volcánico, se identifican 4 unidades litológicas: Cenizas sub- recientes, Formación Sapper y Grupo volcánico del Irazú, Miembro de Lavas Viejas y Terrazas Fluviales (Aluviones) (Arias y Denyer 1992).

Mapa 1. Localización de la cuenca del río Macho en su contexto regional.

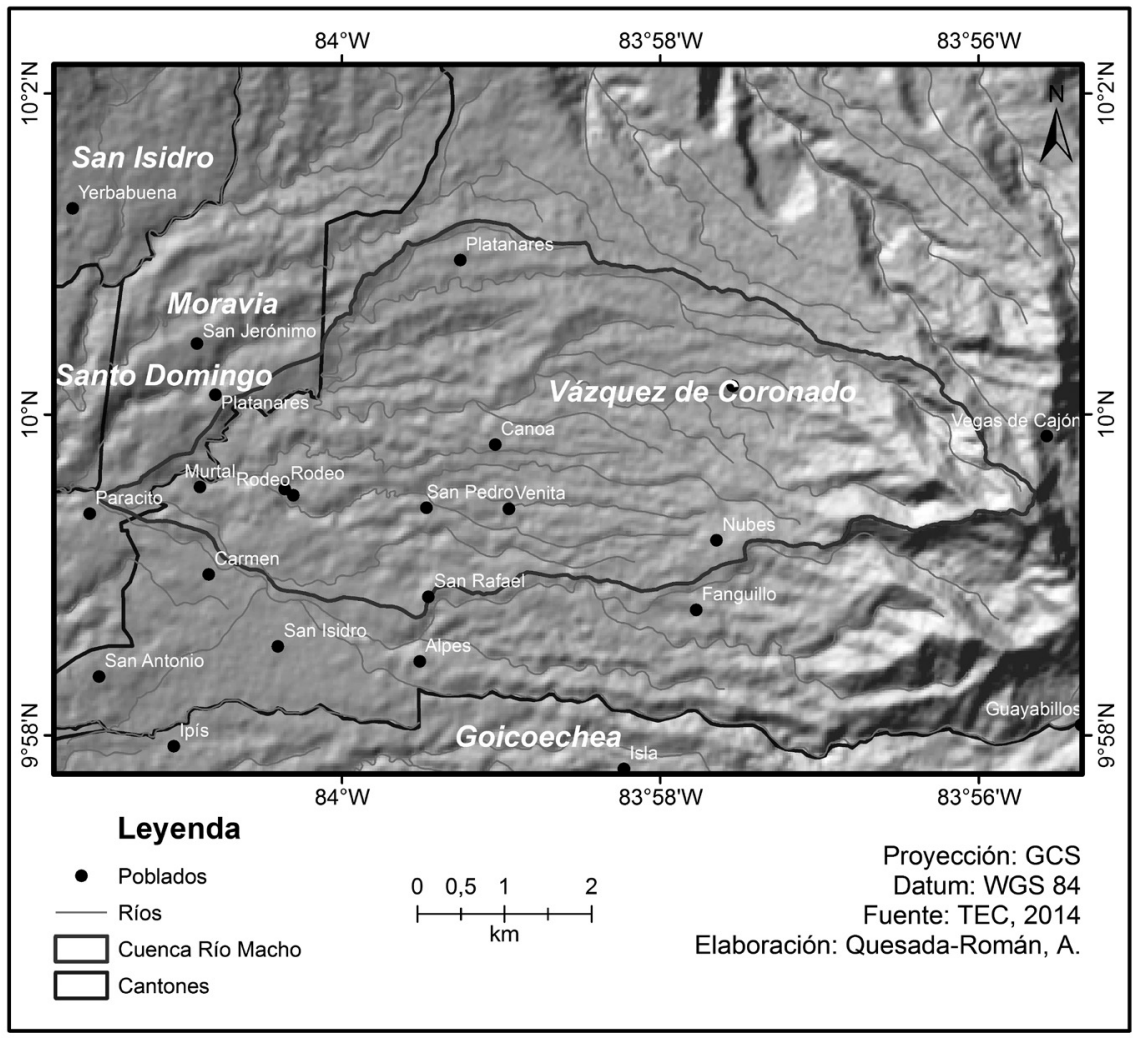


Adolfo Quesada Román, Sergio Feoli Boraschi. A Comparison of the Mora-Vahrson Methodology and the Morphometric Method to Determine Areas Susceptible to Landslides in the Macho River Microbasin, Costa Rica

Las cenizas sub- recientes del Cuaternario conforman la mayor parte del área de la microcuenca, ceniza depositada por la actividad volcánica proveniente de los macizos volcánicos Irazú, Poas y Barva. Las cenizas cubren un $70 \%$ de la totalidad de la microcuenca excepto en aquellos sectores, donde la erosión fluvial permite el afloramiento de otros materiales. Es común observar las capas de ceniza en los cortes de caminos, el color de la ceniza meteorizada es gris y para la ceniza sana es azulado, estos materiales proceden de las erupciones periódicas de piroclastos del volcán Irazú, lográndose diferenciar 4 capas de ceniza de diferentes erupciones (Vargas, 2001). En los sectores cubiertos por ceniza se encuentran suelos fértiles atractivos para las actividades económicas de pastizales y cultivos.

La Formación Sapper y el Grupo volcánico del Irazú, del Pleistoceno, son una secuencia de 15 o más flujos de lava, lahares intertratificados y capas de ceniza que sobreyacen, discordantemente, muy semejantes a la formación Reventado, en el área de la microcuenca, los que se encuentran entre la quebrada Cascajal y el río Durazno son pobres debido a la falta de suficiente profundización de los ríos y por la cobertura de ceniza. La subunidad está compuesta por rocas volcánicas, tales como brechas, lavas, tobas, aglomerados, ignimbritas, ceniza y también muchas corrientes de lodo y lahares.

Por otro lado, el Miembro de Lavas Viejas del Cuaternario a la altura de San Rafael y San Isidro de Coronado se caracteriza por bloques redondeados a semiredondeados de rocas volcánicas, entre ellas, lavas escoriáceas de color rojo, donde los coluvios son menos frecuentes y se caracterizan por bloques de lava que significan un área importante para establecer asentamientos humanos. Por último, las terrazas Fluviales, del Terciario, que se encuentran en la parte baja de la microcuenca en la confluencia del río Macho con el río Virilla, se caracterizan por la importancia de los procesos de erosión sobre los de sedimentación, de forma que el río socava todos sus aluviones, llega al sustrato rocoso y deja sólo algunos de los restos de los aluviones en los bordes del valle. Los aluviones son explotados comercialmente por medio de tajos, en forma de bloques con diámetros mayores de 20 $\mathrm{cm}$, al extraer estos materiales (Denyer y Arias, 1991).

La microcuenca de río Macho, forma parte de una unidad geomorfológica de origen volcánico, la cual se divide en dos subunidades, denominadas Volcán Irazú y Volcán Barva (Denyer y Arias, 1991). Dentro de la 
microcuenca se encuentran definidas seis formas geomorfológicas, entre ellas: barrancas, morfologías con un grado alto de disección, formas de relieve con un moderado grado de disección, relieve alto, áreas de influencia directa con el cauce y planicies aluviales. La unidad geomorfológica de barrancas se encuentra localizada en la parte media alta de la microcuenca del río Macho. Se trata de áreas estrechas a lo largo de las quebradas Honda, Cascajal y Guaba, que han sido formadas por la erosión fluvial o lateral que ejerce sus aguas. Es notable la presencia de fuertes pendientes, por lo que se forman estrechos y profundos encajonamientos o cañones, donde se exponen las rocas de origen volcánico. La presencia de ceniza volcánica en el área de estudio es relevante, porque son materiales poco consolidados y se erosionan con mayor facilidad. Las morfologías con un grado alto de disección son montañas y colinas que son muy irregulares y se pueden encontrar en toda la parte media y alta de la microcuenca, esta sección en la parte alta de la cuenca se caracteriza por tener fuertes pendientes y por estar cubierta de bosque.

Por otro lado, las formas de relieve con un moderado grado de disección se presentan áreas de relieves colinosos y de relieve moderado en cuanto a pendiente. El grado de disección es moderado y los valles fluviales de laderas rectas, tienen entalle profundo con valles en uve simétricos. Los espacios interfluviales son moderados, y las cimas de los cerros son redondeadas. Por su parte, las áreas de influencia directa con el cauce se asocian con la quebrada Piedras y en la desembocadura del río Macho, en la parte baja de la microcuenca, presentan pendiente fuerte en un cauce encañonado y algunas terrazas cercanas al talweg de esta quebrada. La planicie aluvial del río Macho se localiza en la parte baja de la microcuenca, próximo a la desembocadura con el río Virilla y corresponde con una unidad plana, la pendiente generalmente es inferior a los 3 grados, siendo las áreas cercanas a las colinas donde se presenta la mayor pendiente, como resultado de la acumulación de aluvión (Bergoeing y Malavassi, 1982).

Para la variable tipo de suelo en la microcuenca se presentan dos categorías de suelos: andisoles e inceptisoles ándicos, dependiendo de la pendiente; es importante asociar su evolución a partir del grado de meteorización y la predominancia de sus arcillas, pasando de perfiles vítricos cercanos al cráter-origen de los piroclastos, hacia perfiles alofánicos, alofánico (plácido), halloysítico, caolínitico y hasta esmectítico (Alvarado et 
Adolfo Quesada Román, Sergio Feoli Boraschi. A Comparison of the Mora-Vahrson Methodology and the Morphometric Method to Determine Areas Susceptible to Landslides in the Macho River Microbasin, Costa Rica

al., 2014; Mata et al., 2013). En la microcuenca se encuentran dos grupos climáticos: húmedo caliente con estación seca bien establecida, húmedo templado con estación seca moderada, con temperaturas entre 15 y $21^{\circ} \mathrm{C}$, así como precipitaciones entre 2000 y $2800 \mathrm{~mm}$ anuales (Herrera, 1986).

En la microcuenca se encontraron 13 usos diferentes de la tierra: bosque, bosque ripario, pastos arbolados, pastos, tacotales, plantaciones forestales, áreas verdes, distribuidos estos usos principalmente en la zona alta y media de la cuenca. El uso urbano se concentra en la parte baja y está disperso en toda la cuenca como pequeñas manchas de crecimiento, especialmente desarrollos inmobiliarios tipo condominio. Los pastos y pastos arbolados son utilizados para ganadería de leche, que es de uso extensivo e intensivo. Los cultivos se presentan en la parte alta y están representados por cultivos de papa y fresa. Los bosques se encuentran en áreas de riberas de río y en la parte alta de la cuenca, y algunos presentan cierto grado de protección, ya que pertenecen a la Reserva Forestal Cordillera Volcánica Central (Feoli, 2015).

\section{Metodología}

\section{Método Mora-Vahrson}

Mora y Vahrson (1994) describen este modelo como un sistema diseñado para permitir la clasificación de los riesgos de deslizamientos en zonas tropicales sísmicamente activas.

El modelo recibe como entrada cinco factores, los cuales se dividen en dos grupos: susceptibilidad por elementos pasivos (pendiente, litología, humedad del suelo) y susceptibilidad por elementos activos (intensidad sísmica e intensidad de lluvias). Para cada factor, se define un índice de influencia para un determinado sitio y con él, al combinarse de acuerdo a un peso específico ponderado, se obtiene un nivel relativo de amenaza $(\mathrm{H})$, a través de la multiplicación y sumas de los índices por medio de las siguientes ecuaciones.

$$
\mathrm{H}=(\mathrm{Sr} * \mathrm{Sl} * \mathrm{Sh}) *(\mathrm{Ts}+\mathrm{Tp}) \text { Ecuación } 1
$$

En donde:

Pendiente (Sr): representa la topografía del área en estudio. 
El método de Mora y Vahrson (1994), originalmente utilizó datos de relieve relativo, sin embargo, Salazar (2007), posteriormente realizó una variación y cambia esta variable por pendientes y utiliza los rangos propuestos por Van Zuidam (1986), actualmente, con los Sistemas de Información Geográfica (SIG) es más fácil la obtención de estos datos. El Cuadro 1 muestra la clasificación general de la pendiente.

Cuadro 1. Clasificación del factor pendiente según los rangos obtenidos

\begin{tabular}{|c|c|l|l|}
\hline $\begin{array}{c}\text { Rangos de pendientes } \\
\text { (grados) }\end{array}$ & $\begin{array}{c}\text { Peso asignado } \\
\text { (Sr) }\end{array}$ & $\begin{array}{c}\text { Procesos y condiciones } \\
\text { característicos del terreno }\end{array}$ & Colores sugeridos \\
\hline $0-2$ & 1 & Plano o casi completamente & Medio verde oscuro \\
\hline $2-4$ & 2 & Inclinación suave & Verde ligero \\
\hline $4-8$ & 3 & Pendientes inclinadas & Amarillo ligero \\
\hline $8-16$ & 4 & Modelo escarpado & Amarillo anaranjado \\
\hline $16-35$ & 5 & Escarpado & Rojo claro \\
\hline $35-55$ & 6 & Muy escarpado & Rojo oscuro a medio \\
\hline$>55$ & 7 & Extremadamente escarpado & Púrpura oscura \\
\hline
\end{tabular}

Fuente: Van Zuidam (1986)

Litología (SI): este factor representa la influencia de los tipos de materiales, sedimentos y rocas en la activación de los deslizamientos. El Cuadro 2 muestra una clasificación general de diferentes litologías y el peso asignado. 
Adolfo Quesada Román, Sergio Feoli Boraschi. A Comparison of the Mora-Vahrson Methodology and the Morphometric Method to Determine Areas Susceptible to Landslides in the Macho River Microbasin, Costa Rica

Cuadro 2. Clasificación del factor litológico valorando la susceptibilidad a la inestabilidad de las laderas

\begin{tabular}{|l|l|c|}
\hline \multicolumn{1}{|c|}{ Litología } & Clasificación & Factor (SI) \\
\hline $\begin{array}{l}\text { Aluviones: gruesos, permeable, compacto, con nivel freático bajo. } \\
\text { Calizas: duras, permeables. }\end{array}$ & Bajo & 1 \\
$\begin{array}{l}\text { Intrusivos: poco fisurados, bajo nivel freático. } \\
\text { Basaltos, andesita, ignimbritas y similares: sanas, permeables y } \\
\text { poco fisuradas. } \\
\text { Rocas metamórficas: sanas, poco fisuradas, nivel freático bajo. }\end{array}$ & & \\
\hline $\begin{array}{l}\text { Rocas sedimentarias: poco alteradas, estratificación maciza } \\
\text { (decamétrica o métrica), poco fisuradas, nivel freático bajo. } \\
\text { Rocas intrusivas, calizas duras, lava, ignimbritas o metamórficas: } \\
\text { medianamente fisuradas o alteradas, nivel freático o } \\
\text { profundidades intermedias. }\end{array}$ & Moderado & 2 \\
\hline $\begin{array}{l}\text { Rocas sedimentarias, rocas intrusivas, calizas duras, lava ignimbritas, } \\
\text { tobas poco soldadas o metamórficas: medianamente alteradas. } \\
\text { Coluvios, lahares, arenas, suelos regolíticos levemente } \\
\text { compactados: drenaje poco desarrollado, niveles freáticos } \\
\text { relativamente altos. }\end{array}$ & Medio & \\
\hline $\begin{array}{l}\text { Aluviones fluvio-lacustres, suelos piroclásticos poco compactados, } \\
\text { sectores de alteración hidrotermal, rocas fuertemente alteradas } \\
\text { y fracturadas con estratificaciones y foliaciones a favor de la } \\
\text { pendiente, con rellenos arcillosos, niveles fréaticos someros. }\end{array}$ & Alto & \\
\hline $\begin{array}{l}\text { Materiales aluviales, coluviales y regolíticos de muy baja calidad } \\
\text { mecánica: con estado de alteración avanzado, drenaje pobre, se } \\
\text { incluyen las categorías 3 y 4 con niveles freáticos muy someros, } \\
\text { sometidos a gradientes hidrodinámicos muy elevados. }\end{array}$ & Muy alto & \\
\hline
\end{tabular}

Fuente: Mora y Vahrson (1994).

Humedad del suelo (Sh): cuantifica la influencia de la humedad acumulada durante todo el año y puede ser considerada como un punto de partida, ya que las fuertes lluvias pueden actuar como un elemento desestabilizador. Se aplica una metodología simple de un balance hídrico del suelo, la cual requiere solo los valores promedios mensuales. En el paso número uno se utiliza un valor límite de $125 \mathrm{~mm}$, que es representativo de la evapotranspiración potencial media mensual en Centroamérica. Además, se demostró que la infiltración significativa requiere al menos $40 \mathrm{~mm}$ de lluvia acumulada en diez días, lo que corresponde a aproximadamente $125 \mathrm{~mm} /$ mes (Mora y Vahrson, 1994). Se deben seguir los siguientes pasos:

Cada valor de la precipitación media mensual se le asigna un valor de índice, como se muestra en el Cuadro 3. Una vez que se evalúa cada 
mes, el total de los 12 valores mensuales asignados tienen que ser calculados para cada estación pluviométrica analizada. Estos valores totales van de 0 a 24. El total se clasifica en cinco grupos, como se muestra en el Cuadro 4. Luego se realizan interpolaciones de este valor.

Cuadro 3. Clasificación de los valores medios mensuales de las precipitaciones

\begin{tabular}{|c|c|}
\hline $\begin{array}{c}\text { Precipitación media mensual } \\
(\mathbf{m m} / \mathbf{m e s})\end{array}$ & Valor asignado \\
\hline$<125$ & 0 \\
\hline $125-250$ & 1 \\
\hline$>250$ & 2 \\
\hline
\end{tabular}

Fuente: Mora y Vahrson (1994).

Cuadro 4. Factor de humedad resultante de la clasificación de los valores acumulados de los índices promedio de precipitación mensual

\begin{tabular}{|c|c|c|}
\hline $\begin{array}{c}\text { Valor acumulado de los } \\
\text { índices de precipitación }\end{array}$ & Calificación & Factor Sh \\
\hline $0-4$ & Muy bajo & 1 \\
\hline $5-9$ & Bajo & 2 \\
\hline $10-14$ & Medio & 3 \\
\hline $15-19$ & Alto & 4 \\
\hline $20-24$ & Muy alto & 5 \\
\hline
\end{tabular}

Fuente: Mora y Vahrson (1994).

Intensidad sísmica (Ts): este factor se determina mediante el análisis de los deslizamientos de tierra provocados por terremotos para establecer la influencia de intensidades sísmicas. En el Cuadro 5, se muestran los datos de las categorías de influencia utilizando los valores de períodos de retorno de 100 años en relación con los registros históricos. 
Adolfo Quesada Román, Sergio Feoli Boraschi. A Comparison of the Mora-Vahrson Methodology and the Morphometric Method to Determine Areas Susceptible to Landslides in the Macho River Microbasin, Costa Rica

Cuadro 5. Determinación del factor de intensidad sísmica como desencadenante en la generación de deslizamientos de tierra, utilizando valores de intensidad de cien años (Escala Mercalli Modificada), basados en observaciones en Costa Rica y América Central

\begin{tabular}{|c|l|c|}
\hline Intensidades (MM) $\mathbf{T r}=\mathbf{1 0 0}$ años & \multicolumn{1}{|c|}{ Calificación } & Factor Ts \\
\hline Lll & Leve & 1 \\
\hline IV & Muy bajo & 2 \\
\hline V & Bajo & 4 \\
\hline V1 & Moderado & 5 \\
\hline Vll & Medio & 6 \\
\hline Vlll & Considerable & 7 \\
\hline IX & Importante & 8 \\
\hline$X$ & Fuerte & 9 \\
\hline$X 1$ & Muy fuerte & 10 \\
\hline Xll & Extremadamente fuerte & \\
\hline
\end{tabular}

Fuente: Mora y Vahrson (1994).

Intensidad de lluvias (Tp): este factor calcula la influencia de la lluvia en el tiempo, y es otro de los factores desencadenantes que influyen en el disparo de los deslizamientos. En el Cuadro 6, se muestra la clasificación de las precipitaciones máximas diarias para un período de retorno de 100 años.

Cuadro 6. Factor de intensidad de precipitación Tp resultante de la clasificación de las precipitaciones máximas diarias durante un período de retorno de 100 años

\begin{tabular}{|c|c|c|c|}
\hline $\begin{array}{c}\text { Precipitación } \\
\text { máxima n }>\mathbf{1 0} \text { años, } \\
\mathbf{T r}=\mathbf{1 0 0} \text { años }\end{array}$ & $\begin{array}{c}\text { Precipitación media } \\
\mathbf{n}<\mathbf{1 0} \text { años }\end{array}$ & Clasificación & Factor Tp \\
\hline$<100 \mathrm{~mm}$ & $<50 \mathrm{~mm}$ & Muy bajo & 1 \\
\hline $101-200 \mathrm{~mm}$ & $51-90 \mathrm{~mm}$ & Bajo & 2 \\
\hline $201-300 \mathrm{~mm}$ & $91-130 \mathrm{~mm}$ & Medio & 3 \\
\hline $301-400 \mathrm{~mm}$ & $131-175 \mathrm{~mm}$ & Alto & 4 \\
\hline$>400 \mathrm{~mm}$ & $>175 \mathrm{~mm}$ & Muy alto & 5 \\
\hline
\end{tabular}

Fuente: Mora y Vahrson (1994) 
En el Cuadro 6, la columna dos representa una clasificación auxiliar que se basa en los valores medios anuales máximos (aplicable sólo en casos de pluviómetros con registros cortos. De acuerdo con los resultados obtenidos mediante la combinación de todos los parámetros antes mencionados a través de la ecuación 1, el valor del indicador de la amenaza del deslizamiento $(\mathrm{Hl})$ puede ser clasificado y evaluado para cada sitio en particular como se muestra en el Cuadro 7.

Cuadro 7. Clasificación de los valores de los parámetros del peligro de deslizamiento $\mathrm{Hl}$, derivado de la ecuación 1

\begin{tabular}{|c|c|c|}
\hline Valor de la ecuación $(\mathbf{H I})$ & Clase & $\begin{array}{c}\text { Clasificación del peligro } \\
\text { de deslizamiento }\end{array}$ \\
\hline$<6$ & 1 & Muy bajo \\
\hline $7-32$ & 11 & Bajo \\
\hline $33-162$ & 111 & Moderado \\
\hline $163-512$ & lV & Medio \\
\hline $513-1250$ & $\mathrm{~V}$ & Alto \\
\hline$>1250$ & $\mathrm{Vl}$ & Muy alto \\
\hline
\end{tabular}

Fuente: Mora y Vahrson (1994)

Para determinar el índice de susceptibilidad se parte de la ecuación:

$$
H=\left(S_{r} \times S_{1} \times S_{h}\right) \times\left(T_{s}+T_{p}\right)
$$

Se aplicó la propiedad distributiva de la multiplicación, para tener dos mapas de susceptibilidad: uno asociado al factor detonante sismos (Ts) y otro al factor detonante precipitaciones máximas (Tp).

$$
H=\left(S_{r} \times S_{1} \times S_{h} \times T_{s}\right)+\left(S_{r} \times S_{1} \times S_{h} \times T_{p}\right)
$$

El índice de susceptibilidad para la cuenca en cuestión se calculó sumado los dos mapas (Fig. 1). El análisis parte de la ecuación $\mathrm{H}=\mathrm{H}_{\mathrm{s}}+$ $\mathrm{H}_{\mathrm{p}}$, se plantea el siguiente diagrama para iniciar el posterior desarrollo del modelo cartográfico. 
Adolfo Quesada Román, Sergio Feoli Boraschi. A Comparison of the Mora-Vahrson Methodology and the Morphometric Method to Determine Areas Susceptible to Landslides in the Macho River Microbasin, Costa Rica

$$
\begin{aligned}
& \mathrm{H}=\left(\mathrm{S}_{\mathrm{r}} \times \mathrm{S}_{1} \times \mathrm{S}_{\mathrm{h}} \times \mathrm{T}_{\mathrm{s}}\right)+\left(\mathrm{S}_{\mathrm{r}} \times \mathrm{S}_{1} \times \mathrm{S}_{\mathrm{h}} \times \mathrm{T}_{\mathrm{p}}\right) \\
& \mathrm{H}=\text { Grado de susceptibilidad a deslizamientos } \\
& \mathrm{Sr}=\text { Factor pendiente o relieve relativo } \\
& \mathrm{S} 1=\text { Factor litológico } \\
& \mathrm{Sh}=\text { Factor de humedad del suelo } \\
& \mathrm{Ts}=\text { Sismicidad } \\
& \mathrm{Tp}=\text { Intensidad de precipitaciones }
\end{aligned}
$$

Figura 1. Esquema del procedimiento del método Mora-Vahrson

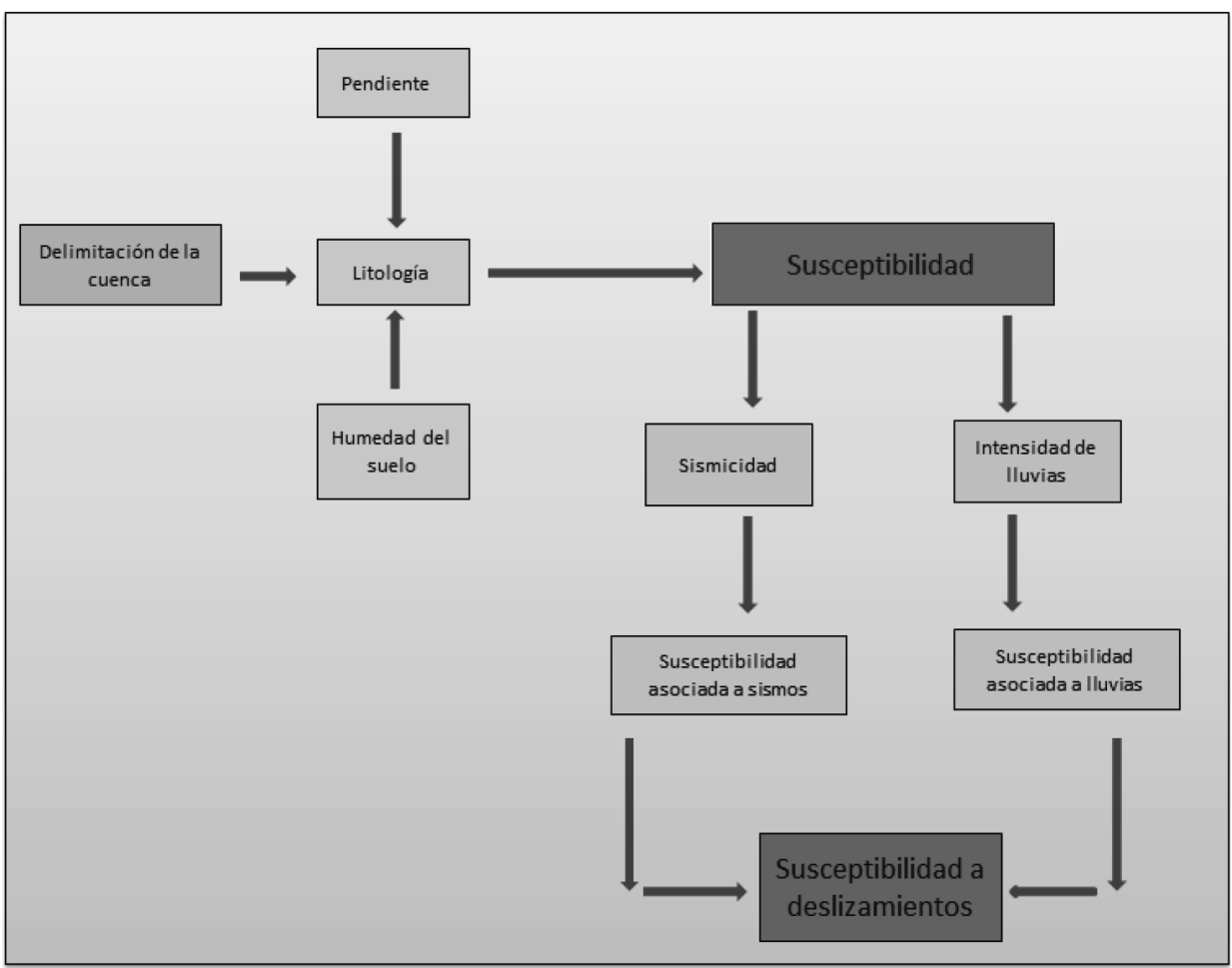

\section{Método morfométrico}

Los métodos morfométricos utilizados se basan en los planteamientos de Simonov (1985), Lugo (1988). Se parte de la utilización de curvas de nivel a escalas superiores a 1:50.000, para este caso a escala 1:25.000 con coordenadas métricas CRTM05. Se seleccionaron cuatro parámetros que representan la dinámica de laderas, derivados del modelo de elevación 
del terreno (MDT) y de la red fluvial, una descripción amplia y completa del modelo puede ser consultada en Quesada y Barrantes (2017).

Las variables utilizadas son la densidad de la disección (D), la profundidad de la disección (P), la energía del relieve (E) y la erosión total (ET). La densidad de la disección (D) calcula la concentración de cauces fluviales en un área específica, tiene como objetivo establecer zonas de mayor o menor concentración de cursos fluviales y por ende con mayor erosión fluvial. Entre las variables que condicionan este parámetro morfométrico resaltan la competencia del sustrato, las estructuras disyuntivas (fracturas o fallas), la inclinación del terreno, la cobertura, así como la intensidad y tipo de precipitación. Se determina trazando todos los cauces fluviales que hay en las cuencas dentro el área de estudio, incluyendo los drenes o torrentes de montaña que no aparecen en la cartografía. Posteriormente, se calcula la concentración de los ríos por unidad de área (unidad mínima de análisis).

La profundidad de la disección (P) tiene como objetivo medir la capacidad o actividad erosiva de los ríos en la vertical, ya que analiza las superficies donde la erosión fluvial ha sido más (o menos) intensa a través del tiempo, y proporciona una relación indirecta de los parámetros que permiten que la disección aumente como la litología, la inclinación del terreno, la precipitación y los planos de debilidad del sustrato. Se explica como la altura entre el talweg y la ruptura de pendiente más representativa y próxima a la ladera inferior del valle (por unidad espacial mínima de análisis) expresada en metros. La energía del relieve (E) determina la diferencia máxima de la altura relativa en metros en un área específica y representa la energía potencial que se desprende del relieve. Para su medición se ubicaron los valores de mayor y menor altitud, en este caso por unidad espacial mínima de análisis, se obtiene la diferencia entre éstos. La erosión total (ET) determina zonas con mayor o menor erosión del relieve registrado por medio de la densidad de las curvas de nivel en un área determinada (por unidad espacial mínima de análisis).

El método morfométrico de susceptibilidad a procesos de ladera se expresa de la siguiente forma: 
Adolfo Quesada Román, Sergio Feoli Boraschi. A Comparison of the Mora-Vahrson Methodology and the Morphometric Method to Determine Areas Susceptible to Landslides in the Macho River Microbasin, Costa Rica

$$
\mathrm{SPL}=\mathrm{D}+\mathrm{P}+\mathrm{E}+\mathrm{ET} / 4[\mathrm{E} 1]
$$

Dónde:

SPL $=$ Susceptibilidad a ocurrencia de procesos de ladera (deslizamientos en su acepción más amplia)

$\mathrm{D}=$ Densidad de la disección

$\mathrm{P}=$ Profundidad de la disección

$\mathrm{E}=$ Energía del relieve

$\mathrm{ET}=$ Erosión total

El modelo ha sido automatizado por medio del uso de Sistemas de Información Geográfica, a través de un modelo que automatiza el cálculo de cada una de las variables morfométricas mediante la herramienta Model Builder de ArcGIS 10 o versiones superiores. Las variables de densidad de la disección, profundidad de la disección, energía del relieve y erosión total fueron calculadas mediante cuadrículas de 200 x $200 \mathrm{~m}$ a partir de curvas de nivel escala 1:25.000 del Catastro Nacional de Costa Rica y los cauces fluviales digitalizados a partir de la cartografía base a escala 1:25.000, así como los cauces que no aparecen y fueron inferidos a partir de las curvas. La rejilla diseñada excede en una celda alrededor de toda el área de estudio, para evitar el efecto de borde en el análisis de interpolación. Todo el procesamiento se realizó por medio de un Sistema de Información Geográfica (SIG), en este caso ArcGIS 10. Al cálculo de cada variable morfométrica realizado para cada celda se le asignó a un punto central de la celda y, posteriormente, se interpoló mediante el método Kriging a partir del modelo de semivariograma ordinario esférico. Todas las variables morfométricas son estandarizadas por medio del método estadístico de Cuartiles, lo que facilita el análisis y distribución de los resultados para la explicación de la dinámica del relieve. Para poder combinar las variables se requiere de una previa estandarización de las mismas, esto se logra con la estandarización omega (ひ) (Velázquez y Celemín, 2011; Buzai, 2013), de esta forma todas las variables se expresan en valores entre 0 y 1 , sin perder información de los valores extremos.

Luego de aplicar la fórmula propuesta [E1], se requiere para establecer clases que representan grados de susceptibilidad a procesos de ladera, se proponen tres clases correspondientes con baja, moderada y alta, dado 
que la metodología propuesta se orienta a su aplicación en el proceso de Gestión del Riesgo, se facilitará su interpretación por parte de los gestores del riesgo y de los tomadores de decisiones (Barrantes-Castillo y Quesada Román, 2016). Adicionalmente, se aclara que la selección del método de clasificación puede ser utilizada para calibrar el modelo, al confrontar el resultado espacial de la clasificación con la distribución del inventario previo de deslizamientos.

\section{Resultados}

\section{Aplicación del método Mora-Vahrson}

La aplicación de la metodología Mora-Vahrson (Mapa 2), muestra que el 35,4\% del área de estudio presenta una susceptibilidad a deslizamientos "Mediana"; mientras que el 8\% del área de la microcuenca tiene una susceptibilidad a deslizamientos "Alta"; la metodología Mora-Vahrson permite realizar zonificaciones de susceptibilidad a deslizamientos, de una forma sencilla por medio del uso de sistemas de información geográfica. La susceptibilidad a deslizamientos "Mediana" está asociada a zonas de alta precipitación y pendientes moderadas (entre $8 \%$ a $30 \%$ ) y geología de origen volcánico y aluvial. La susceptibilidad a deslizamientos "Alta" se presentan en zonas de alta pendiente (más del 30\%), geología de origen volcánica y altas precipitaciones.

Es importante indicar, que se identificaron áreas urbanas (San Rafael, San Pedro, Barrio El Carmen, Paracito) asentadas en zonas de medio y alto grado de susceptibilidad a deslizamientos. Las regiones más susceptibles a deslizamientos se encuentran ubicadas en las partes altas de la microcuenca y se identificaron sitios muy puntuales, principalmente, en los cañones fluviales, donde la susceptibilidad es alta. Estas regiones y las de susceptibilidad moderada pueden ser integradas dentro de la propuesta para el Corredor Biológico Urbano que maneja la Compañía Nacional de Fuerza y Luz. Por otra parte, los factores de humedad en el suelo (Sh), sismos (Ts) e intensidad de precipitaciones (Tp) son valores constantes para toda el área de estudio; por lo que la interacción de los factores pendiente y litología, son los que predominan a la hora de zonificar la susceptibilidad a deslizamientos en esta cuenca. 
Adolfo Quesada Román, Sergio Feoli Boraschi. A Comparison of the Mora-Vahrson Methodology and the Morphometric Method to Determine Areas Susceptible to Landslides in the Macho River Microbasin, Costa Rica

Mapa 2. Microcuenca Río Macho, susceptibilidad a deslizamientos de acuerdo al método Mora-Vahrson, 2017.

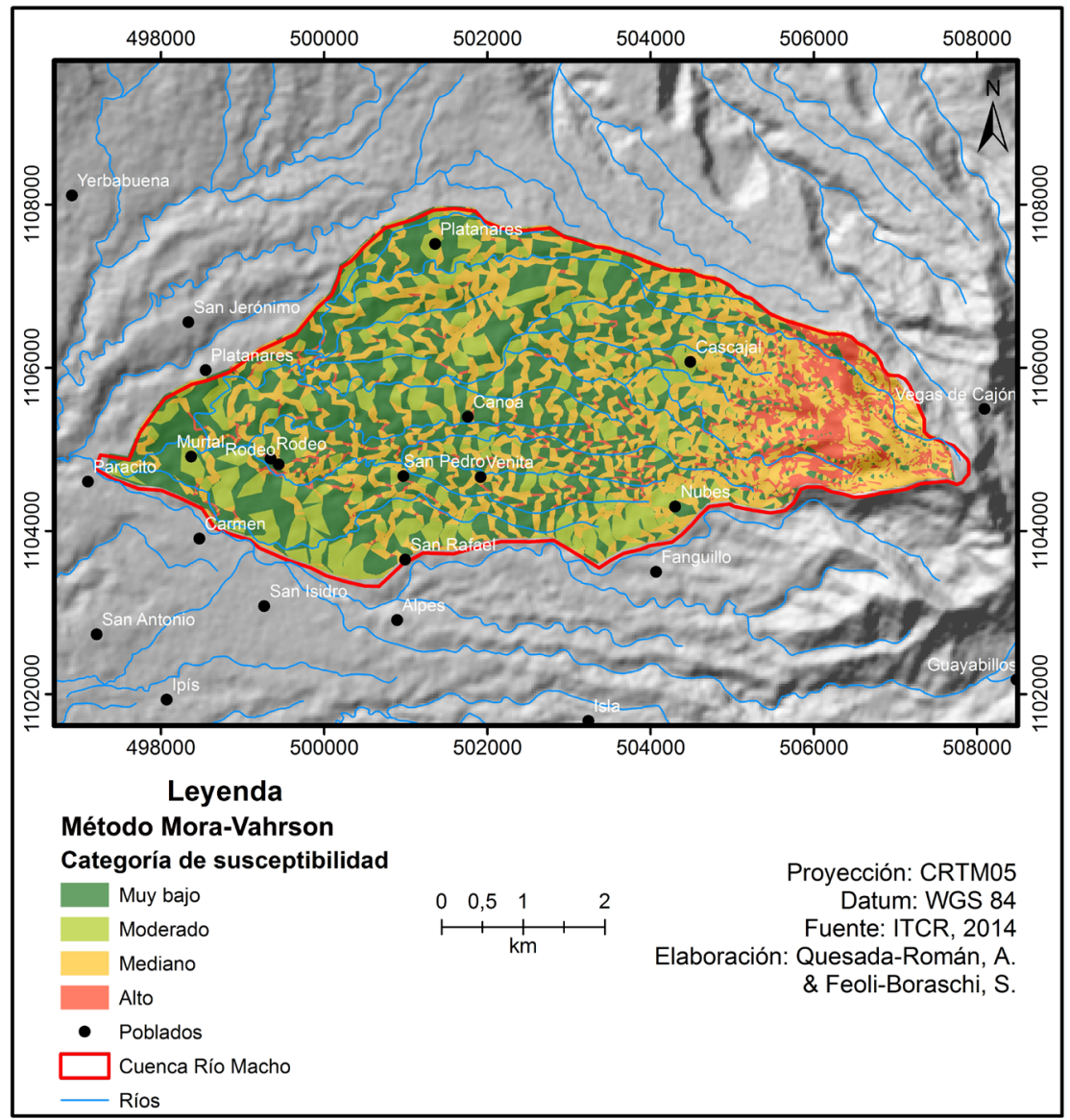

\section{Aplicación del modelo morfométrico}

A partir del análisis de las variables morfométricas utilizadas (densidad de la disección, profundidad de la disección, energía del relieve y erosión total), fue posible determinar tres rangos de susceptibilidad a deslizamientos en la cuenca del río Macho (Mapa 3). Dicha zonificación de estas susceptibilidades a amenazas gravitacionales se asocia directamente a las distintas regiones de la cuenca hidrográfica, como lo son la zona de producción, transición y sedimentación. En las zonas de baja ocurrencia son comunes las pendientes menores a $10^{\circ}$, lo que explica su poca 
incidencia a pesar de localizarse en suelos volcánicos con una alta friabilidad; dichas regiones se podrían asociar más bien a procesos de inundación dada la impermeabilización de los suelos por el desarrollo urbano y las ligeras inclinaciones del terreno.

Las zonas de ocurrencia frecuente se desarrollan en los cañones de los afluentes y el río Macho como lo son las Quebradas Honda, Vuelco, Cascajal, Derrumbe y Palma, las cuales presentan pendientes que se mantienen entre los 10 y $25^{\circ}$. Esta inclinación del terreno favorece la inestabilidad de sus laderas volcánicas aunado a disparadores sísmicos, la influencia climática de la vertiente Caribe, así como la pluviosidad anual por encima de los $2500 \mathrm{~mm}$ y los cambios en el uso de la tierra. Este rango de susceptibilidad también abarca sectores con pendientes mayores a $25^{\circ}$ en las partes altas de la cuenca, donde los materiales volcánicos son aún más friables dada su cercanía con el cráter de emisión del volcán Irazú. Por último, las zonas donde la máxima ocurrencia está presente se localizan de manera aislada en las cabeceras fluviales, con pendientes superiores a los $25^{\circ}$ y además, predominan cauces fluviales no mayores al orden 3 de acuerdo a la clasificación de órdenes de Strahler, con una amplia presencia de suelos andisoles vítricos y alofánicos muy inestables. 
Adolfo Quesada Román, Sergio Feoli Boraschi. A Comparison of the Mora-Vahrson Methodology and the Morphometric Method to Determine Areas Susceptible to Landslides in the Macho River Microbasin, Costa Rica

Mapa 3. Microcuenca Río Macho, susceptibilidad a deslizamientos de acuerdo al método morfométrico, 2017.

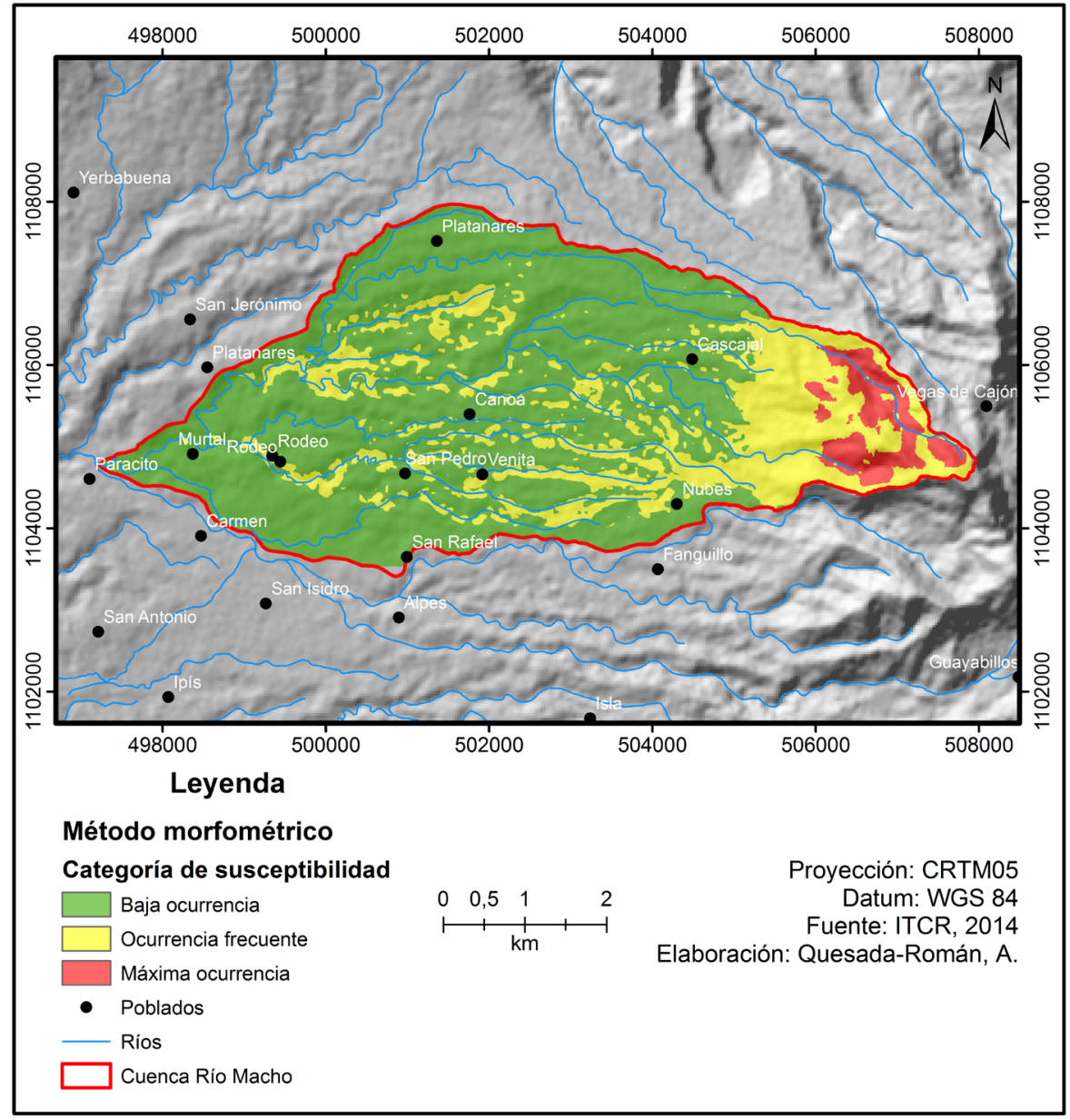

\section{Discusión de resultados}

Los resultados del método Mora-Vahrson responden a las escalas de las variables utilizadas, porque varían de escalas desde 1:50.000, 1:25.000 y 1:10.000, lo que genera uno de los principales problemas a la hora de desarrollar el método y obtener resultados que no reflejan la realidad de los procesos de ladera que ocurren, mayoritariamente, en la parte alta y los valles fluviales. Dado que a veces la información no siempre se encuentra con facilidad o que su adquisición es más complicada, dado el número de 
variables utilizadas, o que se debe digitalizar, representa un aumento de los costos de realización.

Además, realizar mapas de humedad de suelo requiere mucho tiempo, dada la metodología utilizada, según Mora y Vahrson (1994), donde hay que hacer mapas de precipitación por mes y generar el mapa de humedad de suelo para la cuenca, lo que es representativo para cuencas de varias decenas de kilómetros cuadrados, pero no para cuencas pequeñas. Por otro lado, obtener los datos sismológicos es una tarea que conlleva tiempo y dada la tramitología, así como el tratamiento de los datos, donde en países como Costa Rica, existen dos sistemas de monitoreo sísmico, lo que vuelve complejo este análisis, o en el caso de otros países, que esta información pertenece al ejército. Otras de las limitantes es que la obtención de datos geológicos a escalas a detalle es prácticamente nula, ya que se circunscriben a estudios particulares y escalas mayores a 1:50.000, cuando existe. La ventaja de este método recae en que ha sido ampliamente utilizado y es regionalmente conocido por sus resultados rápidos y al mismo tiempo ser importante línea base para posteriores estudios ingenieriles o de planificación del territorio.

La morfometría tiene como ventaja su bajo costo, fácil acceso a la información de base y la rápida obtención de productos cartográficos de calidad y escala. Por medio de este procedimiento, se pueden hacer diagnósticos de base para el ordenamiento territorial de cuencas hidrográficas y unidades político-administrativas de Costa Rica, así como de otros países a escalas locales y regionales. La geomorfometría es una herramienta práctica para la mitigación del riesgo a desastres mediante la determinación de áreas susceptibles a procesos de ladera en general. Este método puede ser replicado con facilidad dado que solamente se utilizan las curvas de nivel y ríos, las mediciones son sencillas y pueden ser realizadas a partir de un Sistema de Información Geográfica en su totalidad. Esta condición agiliza los tiempos de digitalización de cauces fluviales, el procesamiento de la información, la sistematización del procedimiento y la practicidad tanto para hacer distintas mediciones como para la toma de datos puntuales de las isohipsas y fluvios. 
Adolfo Quesada Román, Sergio Feoli Boraschi. A Comparison of the Mora-Vahrson Methodology and the Morphometric Method to Determine Areas Susceptible to Landslides in the Macho River Microbasin, Costa Rica

\section{Conclusiones}

Ambos métodos tuvieron similares resultados en la determinación de las áreas de mayor susceptibilidad a deslizamientos tanto el método Mora-Vahrson en sus zonas de alta y mediana susceptibilidad como en el método morfométrico en sus zonas de ocurrencia frecuente y máxima ocurrencia. No obstante, el método morfométrico se valió únicamente de las curvas de nivel y los cauces de los ríos para determinar las cuatro variables que constituyen su análisis, mientras que el método Mora-Vahrson requiere de cinco factores (pendiente, litología, humedad del suelo, intensidad sísmica e intensidad de lluvias), para desarrollar tanto la susceptibilidad por elementos pasivos como de sus elementos activos.

Para el caso del método Mora-Vahrson, la escala la definiría dos puntos esenciales: el tamaño del área de estudio y la escala de mayor tamaño en los insumos (variables) utilizados en el análisis, además, que las variables deben ser ajustadas a partir de las condiciones geológicas, geomorfológicas y climatológicas de cada región a estudiar. Por su parte la morfometría sirve en todas las escalas dados los datos que utiliza, no obstante, está sujeta a la escala de las curvas de nivel utilizadas, porque de allí derivan los cauces fluviales inferidos ya sea manual o automáticamente. La aplicación futura de uno u otro método en Costa Rica u otras zonas tanto tropicales como templadas puede desarrollarse donde la precipitación y los sustratos meteorizados estén presentes. Los autores de este trabajo consideran que ambas metodologías son útiles para la determinación de línea base para zonificar áreas susceptibles a deslizamientos, lo que a su vez puede ser usado para la gestión del riesgo por movimientos en masa, el ordenamiento, así como la planificación territorial, y su utilización va depender de la disponibilidad de los insumos de entrada de dichos métodos.

\section{Referencias}

Aceves-Quesada, F., López-Blanco, J. y Martín del Pozo, A.L. (2006). Determinación de peligros volcánicos aplicando técnicas de evaluación multicriterio y SIG en el área del Nevado de Toluca, centro de México. Revista Mexicana de Ciencias Geológicas, 23(2), 113-124.

Aceves-Quesada, F., Legorreta-Paulín, G. y Álvarez-Ruiz, Y. (2014). Cartografía geomorfológica para el inventario de procesos gravitacionales en la cuenca endorreica del arroyo La Ciénega, flanco oriental del 
volcán Nevado de Toluca. Boletín de la Sociedad Geológica Mexicana, 66(2), 329-342.

Alcántara-Ayala, I. (2000). Landslides: ¿deslizamientos o movimiento del terreno? Definición, clasificaciones y terminología. Boletín de Investigaciones Geográficas, Instituto de Geografia-UNAM, 41, 7-25.

Alvarado, A., Mata, R. y Chinchilla, M. (2014). Arcillas identificadas en suelos de Costa Rica a nivel generalizado durante el período 19312014: i. historia, metodología de análisis y mineralogía de arcillas en suelos derivados de cenizas volcánicas. Agronomía Costarricense, 38(1), 75-106.

Arias, O. y Denyer, P. (1992). Mapa geológico de la hoja Carrillo Norte (1:50 000). - San José: Instituto Geográfico Nacional.

Barillas, E. (2008). Guía metodológica para la evaluación de zonas susceptibles a deslizamientos disparados por lluvias. Ciudad de Guatemala, Guatemala. Recuperado de: http://www.geociencias.com.gt/ foto/propuesta.pdf

Barrantes, G., Barrantes, O. y Núñez, O. (2011). Efectividad de la metodología Mora - Vahrson modificada en el caso de los deslizamientos provocados por el terremoto de Cinchona, Costa Rica. Revista Geográfica de América Central, 2(47), 141-162.

Barrantes-Castillo, G. y Quesada-Román, A. (2016). Método morfométrico para determinar áreas susceptibles a procesos de ladera en Costa Rica. Póster. II Congreso Centroamericano y del Caribe en Movimientos de Ladera. Tegucigalpa, Honduras. 18 al 20 de julio de 2016.

Bergoeing, J. P. y Malavassi, E. (1981). Carta geomorfológica del Valle Central. Escala 1:50.000. (9 hojas más texto). Instituto Geográfico Nacional, Costa Rica.

Bierman, P.R. y Montgomery, D.R. (2014). Key concepts in geomorphology. W.H. Freeman and Company Publishers.

Borja, R. y Alcántara, I. (2004). Procesos de remoción en masa y riesgos asociados en Zacapoaxtla, Puebla. Investigaciones Geográficas, Boletín del Instituto de Geografía, 53, 7-26.

Borja, R. y Alcántara, I. (2010). Susceptibility to mass movement processes in the municipality of Tlatlauquitepec, Sierra Norte de Puebla. Investigaciones Geográficas, Boletín del Instituto de Geografia, 73, 7-21. 
Adolfo Quesada Román, Sergio Feoli Boraschi. A Comparison of the Mora-Vahrson Methodology and the Morphometric Method to Determine Areas Susceptible to Landslides in the Macho River Microbasin, Costa Rica

Buzai, G. (2013). El mapa social de la ciudad de Luján, 2010. Modelo socioespacial basado en cluster analysis. Tiempo y Espacio, 31, 9-34.

Carignano, C.A., Cioccale, M.A. y Martino, R.D. (2014). El megadeslizamiento del Cerro Uritorco, ladera occidental de la Sierra Chica de Córdoba. Revista de la Asociación Geológica Argentina, 71(1), 21-32.

Cruden, D. M. y Varnes, D. J. (1996). Chapter 3: Landslides Investigation and Mitigation. Landslides types and processes. Transportation Research Board. National Research Council, Special Report, 47.

Dikau, R. (2004). Mass movements. En: Encyclopedia of Geomorphology. Routledge, (A. Goudie, ed.). Taylor \& Francis Group. England. 1156 pp.

Denyer, P. y Arias, O. (1991). Estratigrafía de la región central de Costa Rica. Revista. Geológica de América Central, 12,1-59

Dos Santos, C., Florenzano, T. y Dalla, E. (2009). Estudo geológico-geomorfológico da sub-bacia leste do Araripe com aplicação de variáveis morfométricas derivadas a partir de dados SRTM. Anais XIV Simpósio Brasileiro de Sensoriamento Remoto, Natal, Brasil, 25-30 abril 2009, INPE. 3355-3362.

Evans, I. (2012), Geomorphometry and landform mapping: What is a landform?. Geomorphology, 137, 94-106.

Feoli, S. (2015). Mapa de uso del suelo de las cuencas de la parte alta de la Gran Área Metropolitana. Informe interno para el Departamento Ambiental de la Compañía Nacional de Fuerza y Luz S.A. $76 \mathrm{pp}$. (inédito).

Flores, P. y Alcántara, I. (2002). Cartografía morfogenética e identificación de procesos de ladera en Teziutlán, Puebla. Investigaciones Geográficas, Boletín del Instituto de Geografia, 49, 7-26.

Flores, P. y Alcántara, I. (2012). Susceptibility to shallow landslides of soil in the municipality of Temoaya, Mexico: multicriterial analysis. Investigaciones Geográficas, Boletín del Instituto de Geografía, 77, 31-47.

Galindo, J.A. y Alcántara, I. (2014). Inestabilidad de laderas e infraestructura vial: análisis de susceptibilidad en la Sierra Nororiental de Puebla, México. Investigaciones Geográficas, Boletín del Instituto de Geografia, 88, 122-145.

Herrera, W. (1986). Clima de Costa Rica. Editorial Museo Nacional de Costa Rica 10 mapas, escala 1: 200.000. 
Highland, L. M. y Bobrowsky, P. (2008). The landslide handbook-A guide to understanding landslides. Reston, Virginia, U.S. Geological Survey Circular 1325. 129 pp.

Hervás, J., Barredo, J. y Lomoschitz, A. (2001). Evaluación de la susceptibilidad de deslizamiento mediante el uso conjunto de SIG, Teledetección y métodos de evaluación multicriterio. Aplicación de Tirajana (Gran Canaria). (En línea). Recuperado de: http://eusoils. jrc.ec.europa.eu/library/themes/LandSlides/Documents/Hervas_Barredo_2001_Madrid.pdf

Huggett, R. (2007). Fundamentals of Geomorphology. Routledge. New York, Estados Unidos.

Instituto Meteorológico Nacional (2014). Atlas Climatológico de Costa Rica (CD-ROM). San José Costa Rica. 1 CD-ROM.

INETER (Instituto Nicaragüense de Estudios Territoriales), SNET (Servicio Nacional de Estudios Territoriales) y BGR (Bundesanstalt fuer Geowissenschaften und Rohstoffe). (2004). Mapa de Susceptibilidad a Deslizamientos de Nicaragua: el Método Mora-Vahrson. (En línea). Recuperado de http://webserver2.ineter.gob.ni/geofisica/desliza/estudios/ Mora_Vahrson.pdf

Instituto Tecnológico de Costa Rica, (ITCR). (2014). Atlas digital de Costa Rica. (CD-ROM) Cartago, Costa Rica. 1 CD ROM.

Lal Rajbhandari, P., Monwar, A. y Shakil M. (2002). Application of GIS (Geographic Information System) for landslide hazard zonation and mapping disaster prone area: A study of Kulekhani watershed, Nepal. Plan Plus 1(1), 117-123. Recuperado de http://www.bdresearch. org/home/attachments/article/519/ooo.pdf

Legorreta, G., y Lugo, J.I. (2014). Zonación de peligros por procesos gravitacionales en el flanco suroccidental del volcán Pico de Orizaba, México. Investigaciones Geográficas, Boletín del Instituto de Geografia, 84, 20-31.

Lugo, J. (1988). Elementos de Geomorfología Aplicada (Métodos cartográficos). Instituto de Geografía, UNAM, Ciudad de México, México.

Lugo, J. I., Zamorano, J.J., Capra, L., Inbar, M. y Alcántara, I. (2005). Los procesos de remoción en masa en la Sierra Norte de Puebla, octubre de 1999: causa y efectos. Revista Mexicana de Ciencias Geológicas, 22(2), 212-228. 
Adolfo Quesada Román, Sergio Feoli Boraschi. A Comparison of the Mora-Vahrson Methodology and the Morphometric Method to Determine Areas Susceptible to Landslides in the Macho River Microbasin, Costa Rica

Lugo. J. (2011). Diccionario Geomorfológico. Instituto de Geografía, UNAM. Ciudad de México, México.

Marcano, A. y Cartaya, S. (2013), Zonificación de la amenaza por procesos de remoción en masa originados por las precipitaciones entre Camurí Chico y Punta Tigrillo, estado Vargas, Venezuela. Revista de Investigación, 80(37), 189-214.

Marques, R., Goncalves, F., Conceicao, R., Fernandes, F., Vierira, J.R. y N. Simas (2008). Relief morphometry in the Cachoeira river basin in the management plan of Floresta Nacional de Passa Quatro (MG). R. RA'E GA, Curitiba, 16, 119-128.

Mata R., Rosales A., Vásquez A. y Sandoval, D. (2013). Mapa de Órdenes de Suelos de Costa Rica. 2 ed. San José, Costa Rica. Asociación Costarricense de la Ciencia del Suelo. Escala 1: 200.000. Color.

Mora, R. (2004a). Aplicación de la metodología MVM para determinar la susceptibilidad a deslizamientos en el Bajo Cacao, Atenas, Alajuela. Escuela Centroamericana de Geología. UCR, San José Costa Rica.

Mora, R. (2004b). Evaluación de la susceptibilidad al deslizamiento del cantón de San José, provincia de San José, Costa Rica. Escuela Centroamericana de Geología UCR. San José Costa Rica. Recuperado de http://www. femica.org/areas/modambiental/archivos/foro/ deslizamiento_canton_san_jose.pdf

Mora, R., Chaves, J. y Vázquez, M. (2002). Zonificación de la Susceptibilidad al Deslizamiento: Resultados obtenidos para la Península de Papagayo mediante la modificación del método Mora-Vahrson (Mora, R., Vahrson \& Mora, S., 1992). Servicios Especializados de Laboratorio de Suelos y Rocas, FUNDEVI 0960-00, Vicerrectoría de Investigación 113-A0827, Escuela Centroamericana de Geología, Universidad de Costa Rica.

Mora, R. y Mora, S. (1994). Deslizamientos. En: Denyer, P. \& Kussmaul, K. Atlas geológico de la Gran Área Metropolitana. Editorial Tecnológica de Costa Rica. Cartago. 245-288.

Mora, R., Vahrson, W. y Mora, S. (1992). Mapa de amenaza de deslizamientos, Valle Central, Costa Rica. Centro de Coordinación para la prevención de desastres naturales en América Central (CEPREDENAC). 
Mora, S. y Vahrson, W. (1994). Macrozonation methodology for landslide hazard determination. Bulleting of the Association of Engineering and Geologist, 31(1), 49-58.

Mujica, S. y Pacheco, H. (2013). Metodología para la generación de un modelo de zonificación de amenaza por procesos de remoción en masa, en la cuenca del río Camurí Grande, estado Vargas, Venezuela. Revista de la investigación, 80, 215-243.

Muñiz-Jaúregui, J.A., y Hernández-Madrigal, V.M. (2012). Zonificación de procesos de remoción en masa en Puerto Vallarta, Jalisco, mediante combinación de análisis multicriterio y método heurístico. Revista Mexicana de Ciencias Geológicas, 29(1), 103-114.

Pedraza, J. (1996). Geomorfología: principios, métodos y aplicaciones. Editorial Rueda, Madrid, España.

Peña-Monné, J. L. (1997). Cartografía Geomorfológica: Básica y Aplicada. Geoforma Ediciones, Logroño, España.

Peraldo, G. (2000). Amenaza de deslizamientos, 273-286. En: Denyer, P. y Kussmaul S. (comp). Geología de Costa Rica. Editorial Tecnológica de Costa Rica. Cartago.

Quesada-Román, A. (2016). Peligros geomorfológicos: inundaciones y procesos de ladera en la cuenca alta del río General, Pérez Zeledón, Costa Rica. (Maestría en Geografía con énfasis en Geografía Ambiental). Posgrado en Geografía. Universidad Nacional Autónoma de México. 257 pp.

Quesada-Román, A. y Barrantes-Castillo, G. (2016). Procesos de ladera cosísmicos del terremoto de Cinchona (Costa Rica) del 8 de enero de 2009 (Ms=6,2). Cuadernos de Geografía: Revista Colombiana de Geografía, 25(1), 217-232.

Quesada-Román, A. y Barrantes, G. (2017). Modelo morfométrico para determinar áreas susceptibles a procesos de ladera. Investigaciones Geográficas, Boletín del Instituto de Geografia. E-print. http:// dx.doi.org/10.14350/rig.57318.

Ramírez, R, Santana, G. y Chacón, O. (1996). Mapa de amplificación sísmica del Valle Central, Costa Rica. Revista Geológica de América Central, 19/20, 37-55. 
Adolfo Quesada Román, Sergio Feoli Boraschi. A Comparison of the Mora-Vahrson Methodology and the Morphometric Method to Determine Areas Susceptible to Landslides in the Macho River Microbasin, Costa Rica

Salazar, L. (2007). Modelaje de la amenaza al deslizamiento mediante el sistema de información geográfico ILWIS utilizando el método Mora \& Varhson, 1991. San José Costa Rica. Recuperado de http://hercules. cedex.es/hidraulica/prohimet/Br07/Presentaciones/Salazar.pdf

Segura, G., Badilla, E. y Obando, L. (2011). Susceptibilidad al deslizamiento en el corredor Siquirres-Turrialba. Revista Geológica de América Central, 2(45), 101-121.

Servicio Nacional de Estudios Territoriales (SNET). (2004). Memoria técnica para el mapa de susceptibilidad de deslizamientos de tierra en El Salvador. San Salvador, El Salvador. Recuperado de http://www. snet.gob.sv/Documentos/SusceptMovMasa-ES-SNET-2004.pdf

Simonov, Y. (1985). Análisis morfométrico. Universidad Estatal de Moscú, Lomonosov. Moscú, URSS.

Suárez, A. M., Peraldo, G., Badilla, E. y Obando, L.G. (2009). Zonificación geomorfológica para la evaluación de la susceptibilidad a los deslizamientos en la cuenca del río Viejo, Puriscal, Costa Rica. Revista Geológica de América Central, 41, 55-69.

Vargas, A. (2001). Contribución a la geología y geomorfología del cantón de Coronado; Costa Rica. Revista Geológica de América Central, 24, 67-78.

Vahrson, W. (1992). Distribuciones de los periodos de retorno de tres eventos de lluvias extremas en Costa Rica. Revista Geográfica de América Central, 25-26, 193-207.

Valerio, V., López, C. y Alcántara, I. (2012). Mass movement processes associated with volcanic structures in Mexico City. Investigaciones Geográficas, Boletín del Instituto de Geografía, 79, 48-74.

Van Zuidam, R. A. (1986). Aerial Photointerpretation in terrain analysis and geomorphologic mapping. Ed. Smits Publishers, The Hague.

Velázquez, G. A. y Celemín, J. P. (2011). Elaboración y aplicación de un índice de calidad ambiental para la Región de Cuyo, Argentina, 2010. Cuadernos Geográficos, 49(2), 179-197.

Villacorta, S., Llorente, M., Laín, L., Fidel, L., Machare, J. y Carlotto, V. (2007). Análisis de la susceptibilidad a los movimientos de ladera en la cuenca del río Llaminchán (Cajamarca, Perú). Revista del Instituto de Investigaciones FIGMMG, 19, 103-112. 
Adolfo Quesada Román, Sergio Feoli Boraschi. Comparación de la Metodología Mora-Vahrson y el Método Morfométrico para Determinar Áreas Susceptibles a Deslizamientos en la Microcuenca del Río Macho, Costa Rica

International Geotechnical Societies UNESCO Working Party on World Landslide Inventory (WP/WLI) (1990). A suggested method for reporting a landslide. Bulletin of the International Association of Engineering Geology, 41, 5-12. 